\title{
Estudio del comportamiento termotrópico del DPPC mediante la técnica ESR utilizando spin probe DTBN
}

\section{Study of the thermotropic behavior of DPPC using the ESR technique using} $D T B N$ rotation probe.

María Fernanda Heredia Moyano. ', Sandra Fabiola Heredia Moyano. ${ }^{2}$, Luis Miguel Santillán Quiroga. ${ }^{3}$

\begin{abstract}
.
DOI: https://doi.org/10.33262/cienciadigital.v3i4.920

Electron Spin Resonance (ESR) is an experimental technique for detecting and characterizing chemical system for unpaired electrons. In presence of an external magnetic field, the magnetic moments on different centers tend to align with the field and hence with each other, whereas in the absence of a field, the unpaired electrons are aligned randomly.

Electron magnetic resonance occurs when an electromagnetic wave in the microwave region interacts with the sample immersed in a magnetic field, in quantum mechanics this effect is called Zeeman Effect, which is an energy splitting between the two allowed spin state.

It is important to do a ESR study to prove and make comparisons about what theory says of biologic components characteristics, in this case a lipid molecule.

This study will be developed preparing a solution and a lipid molecule, then they will be studied in the ESR spectrometer, one by one, and together at different temperatures.

\footnotetext{
${ }^{1}$ Escuela Superior Politécnica de Chimborazo, Facultad de Ciencias, Riobamba, Ecuador, mariaf.heredia@espoch.edu.ec

${ }^{2}$ Universidad de la Calabria, Departamento de Química, Rende, Italia, herediafhm@gmail.com

${ }^{3}$ Escuela Superior Politécnica de Chimborazo, Facultad de Ciencias, Riobamba, Ecuador, luis.santillanquiroga@espoch.edu.ec
} 
Also, to verify the theoretic and experimental data, the partition parametric will be used by measuring the intensity of the spectra and using equations at diverse temperatures. The analyses of the obtained data show that a change of conformation in the lipid molecule exists when the temperature is increased and the pre transition and main phase are observed., the equations of maximum rainfall intensities proposed by the INAMHI are used to obtain

Keywords: ESR, DTBN, DPPC, Partition parametric.

\section{Resumen.}

La técnica de espectroscopia Electron Spin Resonance (ESR) es una técnica experimental para detectar y caracterizar sistemas químicos que poseen electrones impares. En presencia de un campo magnético externo, el momento magnético en diferentes centros tienden a alinearse con el campo y además con cada uno de ellos, así como en ausencia del campo los electrones impares son alineados aleatoriamente.

La resonancia magnética del electrón ocurre cuando una onda electromagnética en la región del microondas interactúa con la muestra inmersa en un campo magnético, a este efecto se le llama efecto Zeeman en mecánica cuántica, que es un movimiento energético split entre dos estados permitidos.

Es importante hacer un estudio de ESR para comprobar y hacer comparaciones de lo que nos dice la teoría en cuanto a características de componentes biológicos como en este caso de una molécula lipídica,

Este estudio se realizará preparando una solución y una molécula lipídica y luego serán estudiadas en el espectrómetro ESR por separado y juntas a diferentes temperaturas. Además, para la verificación de los datos teóricos y experimentales se utilizará un parámetro de partición mediante la medición de intensidad del espectro y utilizando ecuaciones, a varias temperaturas. El análisis de los datos obtenidos indica que existe un cambio de conformación de la molécula lipídica al incrementar la temperatura y se observa la fase de pre transición y la transición principal.

Palabras claves: ESR, DTBN, DPPC, Parámetro de partición.

\section{Introducción.}

Las membranas lipídicas pueden ser estudiadas a través de la técnica Electron Spin Resonance ESR, que es una técnica espectroscópica ampliamente usada para determinar el análisis de especies paramagnéticas que contienen al menos un electrón impar.

Existen en la naturaleza sustancias paramagnéticas estables que puede ser aplicadas con la técnica ESR a especies biológicas que no contienen especies radicales, se las puede inserir 
centros paramagnéticos utilizando un spin label como prueba (Chiesa, M., Giamello, E., pp1$3)$.

La espectroscopia Electron Spin Resonance ha encontrado constantemente aplicaciones en la problemática de la bioquímica y biomedicina, como un medio potente y sensible para la revelación de especies con radicales. De modo que se aplica tanto a la identificación de radicales intermedios en reacciones metabólicas y cuanto, a observaciones de especies paramagnéticas estables presentes en la naturaleza, o al levantamiento de radicales producidos por radiaciones externas y también en el análisis de sondas paramagnéticas introducidas en sistemas biológicos específicos (spin labeling).

Este fenómeno se lo conoce con diversos nombres, pero son equivalentes, que tienen la finalidad de enfatizar aspectos diferentes del mismo fenómeno, entre los más comunes están "Resonancia Paramagnética Electrónica” (EPR) ó Resonancia Electrónica de Spin (ESR).

El objetivo de este análisis es estudiar el comportamiento termotrópico de una bicapa de DPPC (dipalmitoilfosfatidilcolina) mediante espectroscopía ESR, en presencia de un spin probe DTBN (diterbutilnitroxide), identificando las temperaturas de pre transición (Tp) y de transición principal de fase $(\mathrm{Tm})$.

\section{Metodologia.}

\section{ESR}

Esta técnica utiliza la propiedad intrínseca del electrón, es decir su propio spin s, que hace que el electrón pueda asociarse con un momento dipolar magnético $\mu$, definido como $\mu=$ $-g \beta \boldsymbol{S}$, donde $g$ es el factor de Landè igual a 2,0023 por electrón libre y $\beta$ es el magnetón de Bohr definido

como

$\beta=\frac{e \hbar}{2 m_{e}}$. Los valores permitidos de energía son dados por $E= \pm \frac{1}{2} g \beta H$ considerando que $m_{s=} 1 / 2$.

En ausencia de un campo magnético $\mathrm{H}$, los espines presentes en la muestra se orientan en forma aleatoria en el mismo estado energético, si se introduce un campo magnético uniforme con una orientación definida $H=(0,0, H)$, es decir a lo largo del eje $z$ y los spines se orientan en la dirección del campo.

La energía de interacción entre $\mu$ y $\mathrm{H}$ está definida por la Hamiltoniana de Zeeman $H_{Z}=$ $-\mu \cdot H$, si el campo magnético está orientado sobre el eje z la expresión resultante es la Hamiltoniana de Interacción de Zeeman $H_{Z}=-\mu_{z} \cdot H=g \beta H \boldsymbol{S}$. 
Entonces en un sistema con $s \neq 0$ y con $s=1 / 2$, la interacción de los dipolos con el campo magnético estático nos lleva a la formación de dos niveles energéticos, en el cual los dipolos se orientan como vemos en la figura 1.

Figura 1: Niveles energéticos de Zeeman

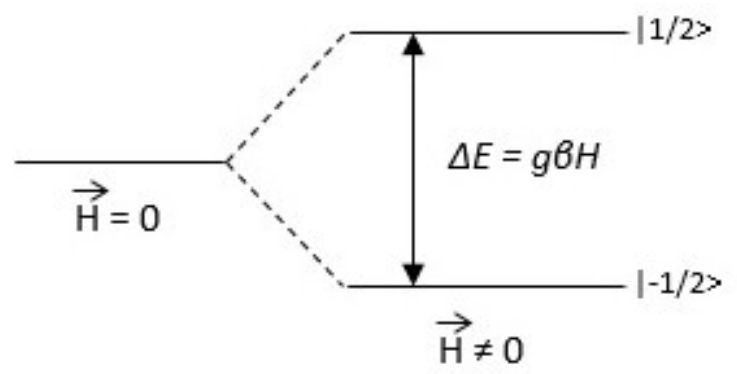

Fuente: Elaboración Propia

\section{Comportamiento termotrópico}

Los liposomas son vesículas fosfolipídicas con un diámetro variable entre $5 \mathrm{~nm}$ y $1 \mu \mathrm{m}$, y están normalmente constituidas por una doble capa de fosfolípidos entre otros componentes como puede ser también el colesterol.

Al aumentar la temperatura a las moléculas lipídicas, estas sufren un cambio conformacional o una transición de fase al absorber el calor, se puede estudiar el cambio de un estado GEL (estado ordenado) a un estado FLUIDO (estado desordenado).

A bajas temperaturas el DipalmitoilfosfatidilcolinaColina (DPPC) se encuentra en un estado ordenado, en fase GEL $L_{\beta}$, en el cual las cadenas están en la misma conformación all-trans, se mueven muy poco y presentan una inclinación de $36^{\circ}$ respecto a la bicapa. Si se aumenta la temperatura en alrededor de los $32^{\circ} \mathrm{C}$ y la bicapa conserva su fase GEL, pero presenta una ondulación en la superficie, la cual toma el nombre de fase RIPPLE $P_{\beta}$, a esta fase se la conoce también como PRE-TRANSICIÓN.

Aumentando aún más la temperatura alrededor de $\operatorname{los} 41^{\circ} \mathrm{C}$ se llega al estado líquido cristalino, es decir la fase fluida, desordenada $L_{\alpha}$. En esta fase las cadenas son desordenadas y el área de la cabeza polar del lípido aumenta.

\section{Coeficiente de Partición}

Para valorar la concentración de prueba en la región hidrofóbica del DPPC usamos el coeficiente de partición $P=\frac{h_{l}}{h_{l}+h_{w}}$, en donde $h_{l}$ es la concentración de spin probe de los lípidos, mientras $h_{w}$ es la concentración de spin probe en agua.

\section{Preparación de la muestra}

Se preparó una solución de Diterbutilnitroxilo DTBN $10^{-3} \mathrm{M}$ y $5 \times 10^{-4} \mathrm{M}$ en agua destilada. La concentración de DTBN $5 \times 10^{-4} \mathrm{M}$ se centrifuga y se la mide en el espectrómetro ESR a una potencia de $10 \mathrm{~mW}$, con una frecuencia de modulación del campo de $100 \mathrm{kHz}$ y una 
amplitud de modulación de 025 Gauss. Se realizaron medidas del espectro de resonancia del campo a temperaturas de $25^{\circ} \mathrm{C}, 35^{\circ} \mathrm{C}$ y $50^{\circ} \mathrm{C}$.

Se preparó la muestra de DPPC, disolviendo $7.35 \mathrm{mg}$ de polvo del DPPC en cloroformo y se la evaporó bajo un flujo de nitrógeno gaseoso, se obtuvo finalmente una película lipídica al cual se deja reposar en el vacío por 24 horas. Se hidrata la película de DPPC con la solución de DTBN $5 \times 10^{-4} \mathrm{M}$ en agua destilada a $50^{\circ} \mathrm{C}$, se insertó en un capilar para ser medido en el espectrómetro de ESR.

Las medidas del espectro del DTBN en la miscela de DPPC en agua se midieron a las temperaturas de $25^{\circ} \mathrm{C}, 27^{\circ} \mathrm{C}, 30^{\circ} \mathrm{C}, 32^{\circ} \mathrm{C}, 35^{\circ} \mathrm{C}, 37^{\circ} \mathrm{C}, 39^{\circ} \mathrm{C}, 41^{\circ} \mathrm{C}, 43^{\circ} \mathrm{C}, 45^{\circ} \mathrm{C}$ e $50^{\circ} \mathrm{C}$.

\section{Resultados}

En la Figura 2 se muestra la sobre posición del espectro del DTBN en tres diferentes temperaturas $25^{\circ} \mathrm{C}, 35^{\circ} \mathrm{C}$ y $50^{\circ} \mathrm{C}$ y podemos ver que, variando la temperatura, la intensidad del espectro no cambia.

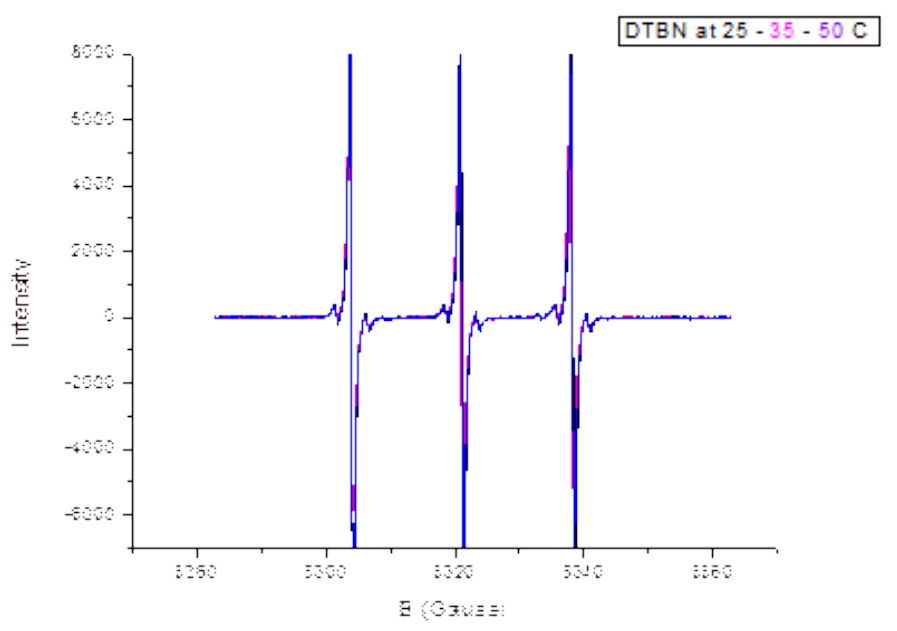

Figura 2. DTB en tres diferentes temperaturas en función del campo magnético Fuente: Elaboración propia.

El DTBN en DPPC en solución acuosa a temperatura $25^{\circ} \mathrm{C}$ y $50^{\circ} \mathrm{C}$ en la fase GEL y la fase LIQUIDO CRISTALINA se puede observar en la Figura 3 la sobre posición de los espectros para evidenciar las variaciones 


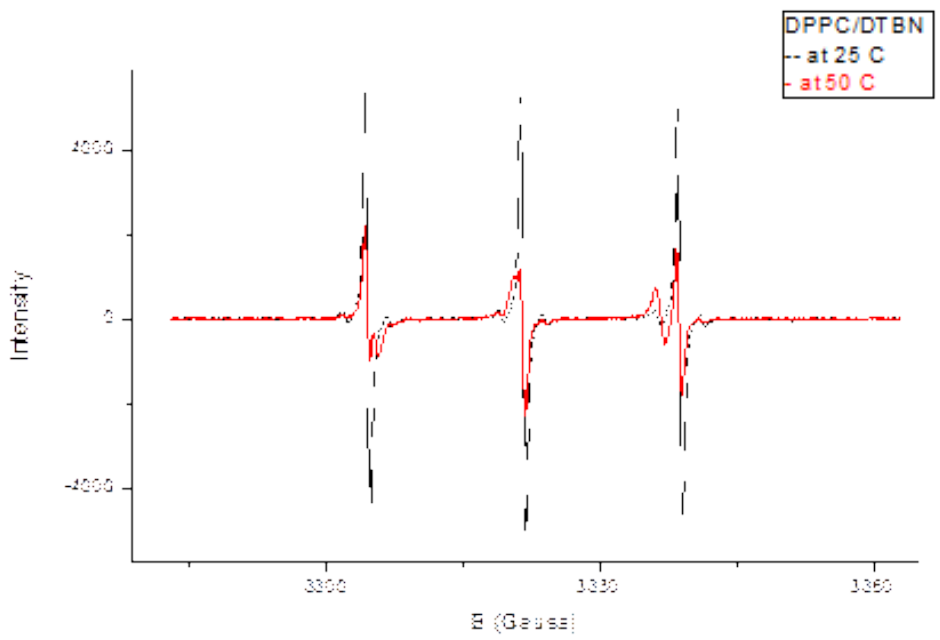

Figura 2. DPPC/DTBN a $25 \stackrel{\circ}{\circ}$ en negro y línea cortada y el DPPC/DTBN a $50 \stackrel{\circ}{\mathrm{C}}$ en rojo y línea continua.

Fuente: Elaboración propia.

Sobreponiendo finalmente los espectros del DTBN en DPPC a la temperatura de pretransición $\mathrm{T}_{\mathrm{p}}$ de $35{ }^{\circ} \mathrm{C}$ y a la temperatura de la transición principal $\mathrm{T}_{\mathrm{m}}$ de $41{ }^{\circ} \mathrm{C}$ como podemos ver en la Figura 4, en donde verificamos la variación en la intensidad del espectro que disminuye una vez que el DTBN interacciona con el DPPC.

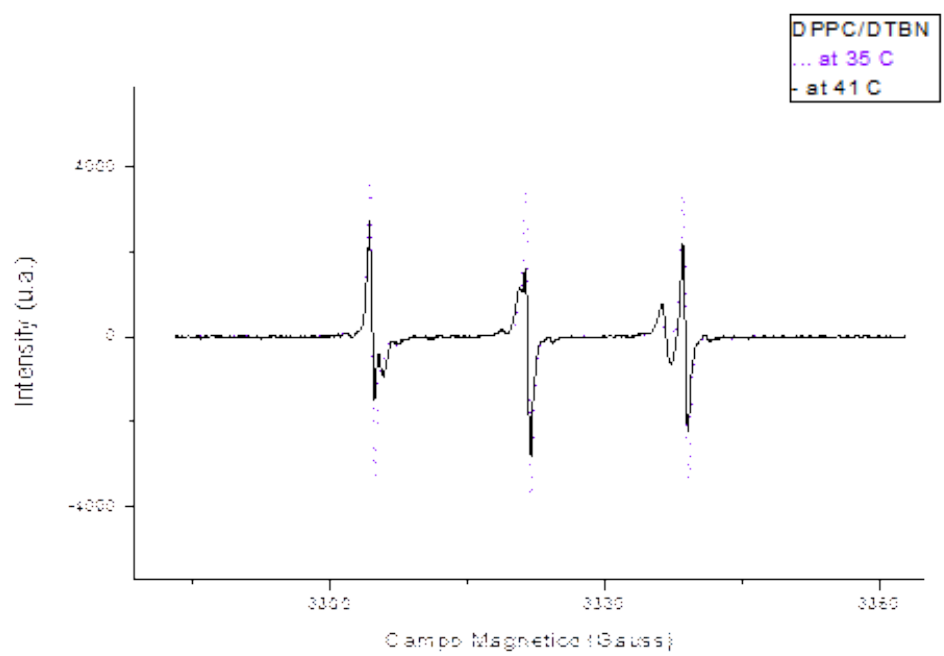

Figura 3. DPPC/DTBN a temperatura de pre transición $35^{\circ} \mathrm{C}$ y la transición principal a $41^{\circ} \mathrm{C}$ Fuente: Elaboración propia.

A través de los espectros de la muestra de DTBN en DPPC registrados a varias temperaturas, se puede medir la altura $h_{1} y h_{w}$, para poder calcular el parámetro de partición P y el parámetro corregido Pc*, los cuales podemos ver en la Tabla 1. 
Tabla 1. Valores de $\mathrm{HL}, \mathrm{HW}, \mathrm{Pc}$ y $\mathrm{Pc}^{*}$ en varias temperaturas.

\begin{tabular}{ccccc}
\hline Temperatura ${ }^{\circ} \mathrm{C}$ & HL & HW & Pc & Pc* \\
\hline 25 & 195,25 & 4753,836 & 0,03945 & 0,13099 \\
27 & 230,213 & 4574,626 & 0,04791 & 0,1559 \\
30 & 212,457 & 4178,573 & 0,04838 & 0,15726 \\
32 & 239,782 & 4189,714 & 0,05413 & 0,17358 \\
35 & 305,134 & 3535,575 & 0,07944 & 0,24055 \\
37 & 338,856 & 3245,185 & 0,09454 & 0,27705 \\
39 & 336,9761 & 3135,14 & 0,09705 & 0,28288 \\
41 & 756,497 & 2205,29 & 0,25767 & 0,55732 \\
43 & 723,706 & 2080,664 & 0,25806 & 0,57562 \\
45 & 714,094 & 2002,535 & 0,26286 & 0,57937 \\
50 & 678,084 & 1740,176 & 0,28041 & 0,58489
\end{tabular}

Los valores de Pc* nos ayuda a estudiar el cambio en función de la temperatura, en la Figura 5, y se hace una comparación entre la curva experimental con la distribución de Pc* teóricos.

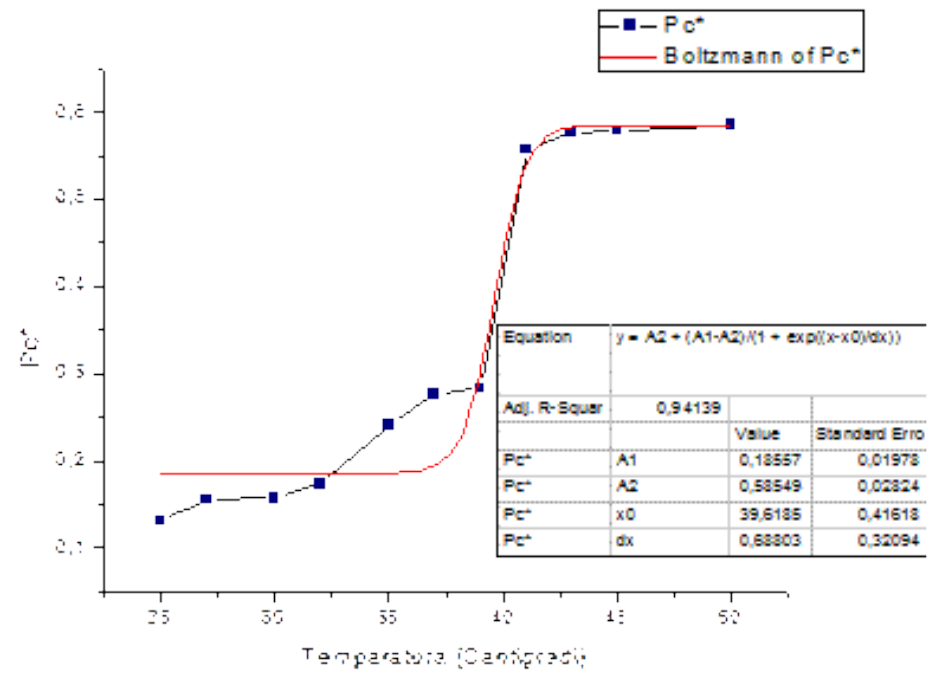

Figura 4. Coeficiente de partición corregido $\mathrm{Pc}^{\star}$ en función de la temperatura.

Fuente: Elaboración propia. 


\section{Conclusiones.}

- Se puede hacer el estudio del cambio de transición de fase de moléculas biológicas a través de técnicas de espectrometría, como en este caso se usó el Electron Spin Resonance ESR.

- Se comprueba lo que nos dice la teoría que, al cambiar de temperatura a una membrana lipídica, esta pasa de un estado ordenado a un estado desordenado, habiendo así un cambio de fase.

- Se verifica el cambio del espectro en la mitad cuando el DTBN interacción con el DPPC, lo cual quiere decir que la bicapa permite el ingreso del DTBN al aumentar la temperatura.

- De la comparación de la curva teórica y experimental del Pc* en función de la temperatura se puede notar que hay una similitud en la zona principal de transición.

\section{Referencias bibliográficas.}

Balatsky, A., Nishijima, M., and Manassen, Y., (2011). Electron spin resonance-scanning tunneling microscopy. Online: https://www.tandfonline.com/doi/abs/10.1080/00018732.2012.668775

Bartucci, R., Mollica, P., and Sportelli, L., (1998). Procain interaction with DPPC multilayers: an ESR spin label investigation. Online: https://link.springer.com/article/10.1007\%2FBF03161870.

Bóta, A., et al. (2000). Strutural behaviours of DPPC/water liposomes.

Cook, R., and Whiffen, D., (2016). Electron spin resonance and its applications. Online: https://iopscience.iop.org/article/10.1088/0031-9155/7/3/201.

Chiesa, M., and Giamello, E., (2000). Electron Spin Resonance Spectroscopy. Online: https://onlinelibrary.wiley.com/doi/abs/10.1002/9780470027318.a6104.pub2.

Escudero, R., et al. (2019). Electron spin resonance (ESR) spectroscopy study of cheese treated with accelerated electrons. Online: https://www.sciencedirect.com/science/article/pii/S0308814618316728.

Fei, K., el at. (2018). Nanoscale zero-field electron spin resonance spectroscopy. Online: https://www.nature.com/articles/s41467-018-03969-4

Kinoshita, A., Baffa, O., and Mascarenhas. S., (2018). Electron spin resonance (ESR) dose measurement in bone of Hiroshima A-bomb victim. Online: https://journals.plos.org/plosone/article?id=10.1371/journal.pone.0192444. 
Mallard, J., and Kent, M. (2016). Electron spin resonance in biological tisues Online: https://iopscience.iop.org/article/10.1088/0031-9155/14/3/201/meta.

Qin, P., Warncke, K., (2015). Electron Paramagnetic Resonance Investigations of Biological Systems by Using Spin Labels, Spin Probes, and Intrinsic Metal Ions. Online: https://www.ncbi.nlm.nih.gov/pmc/articles/PMC6171503/

Shunsuke, C., Tsutomu, M., Electron spin resonance for the detection of long-range spin nematic order.

Online: https://journals.aps.org/prb/abstract/10.1103/PhysRevB.97.104411 
PARA CITAR EL ARTÍCULO INDEXADO.

Heredia Moyano, M., Heredia Moyano, S., \& Santillán Quiroga, L. (2019). Estudio del comportamiento termotrópico del DPPC mediante la técnica ESR utilizando spin probe DTBN. Ciencia Digital, 3(4), 34-43. https://doi.org/10.33262/cienciadigital.v3i4.920

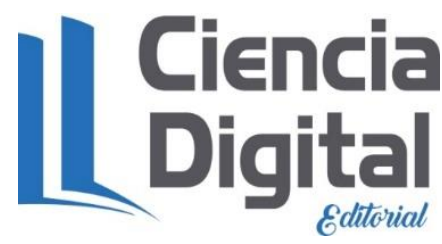

El artículo que se publica es de exclusiva responsabilidad de los autores y no necesariamente reflejan el pensamiento de la Revista Ciencia Digital.

El artículo queda en propiedad de la revista y, por tanto, su publicación parcial y/o total en otro medio tiene que ser autorizado por el director de la Revista Ciencia Digital.
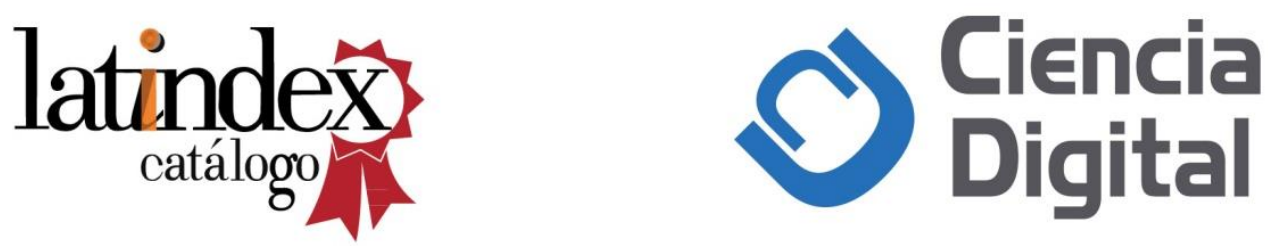